\title{
Is That within Reach? fMRI Reveals That the Human Superior Parieto-Occipital Cortex Encodes Objects Reachable by the Hand
}

\author{
Jason P. Gallivan, ${ }^{1}$ Cristiana Cavina-Pratesi, ${ }^{3}$ and Jody C. Culham ${ }^{1,2}$ \\ ${ }^{1}$ Neuroscience Program and ${ }^{2}$ Department of Psychology, University of Western Ontario, London, Ontario, Canada N6A 5C2, and ${ }^{3}$ Department of \\ Psychology, Durham University, Durham DH1 3LE, United Kingdom
}

\begin{abstract}
Macaque neurophysiology and human neuropsychology results suggest that parietal cortex encodes a unique representation of space within reach of the arm. Here, we used slow event-related functional magnetic resonance imaging (fMRI) to investigate whether human brain areas involved in reaching are more activated by objects within reach versus beyond reach. In experiment 1, graspable objects were placed at three possible locations on a platform: two reachable locations and one beyond reach. On some trials, participants reached to touch or grasp objects at the reachable location; on other trials participants passively viewed objects at one of the three locations. A reach-related area in the superior parieto-occipital cortex (SPOC) was more activated for targets within reach than beyond. In experiment 2, we investigated whether this SPOC response occurred when visual and motor confounds were controlled and whether it was modulated when a tool extended the effective range of the arm. On some trials, participants performed grasping and reaching actions to a reachable object location using either the hand alone or a tool; on other trials, participants passively viewed reachable and unreachable object locations. SPOC was significantly more active for passively viewed objects within reach of the hand versus beyond reach, regardless of whether or not a tool was available. Interestingly, these findings suggest that neural responses within brain areas coding actions (such as SPOC for reaching) may reflect automatic processing of motor affordances (such as reachability with the hand).
\end{abstract}

\section{Introduction}

In primates, parietal cortex includes a variety of areas specialized for actions with different effectors (Andersen, 1997). Such regions include the medial intraparietal area, MIP (which overlaps with the parietal reach region, PRR), visual area V6A, and area 5, involved in arm movements (Colby and Duhamel, 1991; Galletti et al., 2003; Evangeliou et al., 2009), as well as the anterior intraparietal area (AIP) involved in hand movements (Sakata et al., 1992). Each effector can operate only within the range of space specified by its anatomical constraints. For instance, reaching can only be performed to targets within the range of the arm. This space in which the body's effectors can act is termed peripersonal space (Rizzolatti et al., 1985; Previc, 1998) and may be particularly relevant for the visuomotor areas of the brain. Indeed, studies with parietal patients exhibiting extinction (di Pellegrino et al., 1997; Làdavas et al., 1998a,b, 2000) and neglect (Halligan and Marshall, 1991; Berti and Frassinetti, 2000), as well as studies with neurologically intact individuals (Weiss et al., 2000; Makin et al.,

Received Jan. 23, 2009; revised Feb. 24, 2009; accepted Feb. 27, 2009.

This work was supported by an operating grant from the Canadian Institutes of Health Research to J.C. (Grant MOP84293). We are grateful to Jacqueline Snow for her comments on this manuscript. We also thank Joy Williams, Joe Gati, and Teresa McAdam for assistance with fMRI data collection, Adam McLean for software support, and Jim Ladich and Haitao Yang for assistance with hardware development.

Correspondence should be addressed to Jody C. Culham, Department of Psychology, University of Western Ontario, London, Ontario, Canada N6A 5C2. E-mail: jculham@uwo.ca.

D01:10.1523/JNEUROSCI.0377-09.2009

Copyright $\odot 2009$ Society for Neuroscience $\quad$ 0270-6474/09/294381-11\$15.00/0
2007; Quinlan and Culham, 2007), suggest that parietal cortex may preferentially encode near space.

In humans, one area that may be expected to encode peripersonal space is the superior parieto-occipital cortex (SPOC). Functional magnetic resonance imaging (fMRI) has implicated SPOC in both reaching (de Jong et al., 2001; Prado et al., 2005) and pointing preparation (Astafiev et al., 2003; Connolly et al., 2003). Moreover, SPOC may encode not just arm actions per se, but also the intention to make an arm action, as indicated by its response to reachable three-dimensional objects even when no reach is performed (Cavina-Pratesi et al., 2006b). If so, these responses may be modulated by whether or not the object is within reachable space. In addition, responses to reachable objects in SPOC may be modulated by tool use.

Interestingly, Iriki and colleagues (1996), recording from neurons in the anterior bank of the intraparietal sulcus in macaques, found that visual responses originally selective for food items within reach of the arm expanded to encompass the effective range of a tool (rake) after training. Similar effects have been observed in human parietal patients, whose extinction can be recovered in far space after tool use (Farnè and Làdavas, 2000; Maravita and Iriki, 2004). One interpretation of both the macaque and human results is that parietal receptive fields tuned to the arm expand to encompass a tool (Iriki et al., 1996); however, other interpretations exist (Holmes et al., 2004, 2007).

Here, we conducted two experiments using fMRI to investigate the coding of peripersonal space in parietal cortex. First we performed a preliminary experiment suggesting that SPOC may 
show greater responses to reachable versus unreachable objects (Culham et al., 2008). We then conducted a second experiment with rigorous visual and motor controls to test the hypothesis that SPOC encodes reachable space. In addition, we also examined whether the response to reachable locations was modulated by tool use.

\section{Materials and Methods}

Experiment 1: identification of regions tuned to reachable space

The goal of this study was to identity human parietal brain areas involved in hand actions that are tuned to potentially actable object locations (i.e., within reach of the arm). To give participants knowledge about the extent of their reach and to localize areas involved in hand actions, we had them perform reaching and grasping actions toward objects placed on a platform. To measure brain activation corresponding to the potential for action upon reachable objects, on separate trials we had participants passively view objects at both reachable and unreachable locations.

We chose to compare the responses to reachable versus unreachable locations under conditions of passive viewing because other possible tasks, such as reaching, pantomimed reaching, or pointing are problematic. First, actual reaching actions, in which the participant reaches out to touch the target, cannot be performed on objects in unreachable locations by definition. Second, pantomimed or attempted reaching, in which the participant reached toward the object, would have the obvious confound that participants would not succeed for objects beyond reach. Moreover, a past study from our lab has found that pretend actions recruit different brain regions than realistic actions (Króliczak et al., 2007). Third, pointing actions, in which the participant directs the index finger toward the object without actually touching it, can be performed to objects at any distance; thus, there would be no reason to expect any distance-selectivity. Although numerous fMRI studies have examined pointing actions (DeSouza et al., 2000; Astafiev et al., 2003; Connolly et al., 2003; Medendorp et al., 2005), to date reaching and pointing have not been directly compared and may rely on different neural mechanisms. In sum, we were looking for a task that may be expected to be modulated by reachability without explicitly involving reaching or pointing. Passive viewing responses have been observed in both macaque (Sakata et al., 1995) and human brain areas (Cavina-Pratesi et al., 2007) involved in hand actions. These responses are thought to reflect the potential for actions on objects, which as we test here, might be modulated by distance and reachability.

Participants. fMRI was used to measure the blood oxygenation leveldependent (BOLD) signal, which provides a measure of local neural activity (Kwong et al., 1992; Ogawa et al., 1992) from 10 right-handed participants ( 4 males; mean age, 28 years) recruited from the University of Western Ontario (London, Ontario, Canada). Informed consent was obtained in accordance with procedures approved by the university's Health Sciences Review Ethics Board. All participants were naive with respect to the experimental hypothesis and were only informed of the required experimental tasks.

Experimental setup and apparatus. Participants were required to perform action trials (grasping and reaching) and passive viewing trials toward target stimuli placed at different spatial locations upon an angled black platform saddled over each participant's hips (Fig. $1 B$ ). The target stimuli were made of pieces of LEGO and were $\sim 5 \times 2 \times 1.5 \mathrm{~cm}$ in length, depth, and height, respectively. Participants were placed in the bore of the magnet in a supine semireclined position (head tilted $\sim 30^{\circ}$ ) so that the natural line of gaze was directly down the bore of the magnet (toward the feet). This permitted target objects to be presented directly in the workspace in front of the participant without the need of a mirror which would require further spatial transformations and processing. To tilt the head, each participant had their head supported by pieces of foam within the head coil.

Experimental paradigm. An event-related paradigm was used in which each trial lasted $16 \mathrm{~s}$ and consisted of a cue period ( $2 \mathrm{~s}$, during which an auditory instruction was presented to the participant through headphones), a preparation period (6s), an action period [ $2 \mathrm{~s}$, during which participants responded to a stimulus that was illuminated via an light emitting diode (LED)], and a final intertrial interval (6 s) (Fig. $1 A$ ). Participants were instructed to always maintain their gaze on a fixation point (situated along the sagittal midline above a position between the medium and far locations) during the entire length of a run. The fixation point was a green LED positioned $\sim 100 \mathrm{~cm}$ from the participants' eyes and at a visual angle of $16^{\circ}$ above the participants' natural line of gaze. We did not use an eye tracking system during scanning so eye movements could not be monitored.

The auditory cue gave one of three possible instructions: "grasp," "reach," or "look." Grasping trials involved using a precision grip (between thumb and index finger) to manually pick-up (grasp and lift) the object at the medium location. The reaching condition required that the participant reach toward the object at the medium location without preshaping the hand and manually touch the stimuli with the knuckles. The passive viewing ("look") condition involved simply viewing the location of the illuminated object while fixating the LED. Participants performed actions with the right hand and had the right upper arm braced such that arm movement was limited to the elbow and wrist. Movements of large masses such as the shoulder and upper arm have been shown to cause 
perturbations in the magnetic field which induce artifacts in the participant's data (Culham, 2006). The use of an event related paradigm temporally decouples the artifact created from grasping and reaching from the BOLD signal time course (Culham et al., 2003).

The participant was expected to retain the given auditory instruction over the preparation period and perform the cued action with the right hand (for grasping and reaching trials) during the action period when a single object was illuminated. Except for the brief period of object illumination and the fixation LED staying on for the duration of each trial, the magnet bore was completely dark throughout each run. Each participant placed the right hand at a starting position on the left side of the platform in between action trials and during passive viewing trials.

Task. To first explore our hypothesis that SPOC visually encodes reachable objects, we used a relatively simple design where on any given trial a single LEGO object was placed by the experimenter at one of three prespecified locations during the intertrial interval on the angled platform positioned in front of the participant. For each trial, the experimenter randomly selected from eight possible LEGO stimuli. The near location was $\sim 2 \mathrm{~cm}$ from the participant's right hand in the participant's left visual field, the medium location was $\sim 25 \mathrm{~cm}$ from the participant's right hand in their right visual field, and the far location was placed $\sim 55$ $\mathrm{cm}$ from the participant's midline. Most importantly, whereas the stimuli placed within both the near and medium locations were within reach of the participant's braced arm/hand, stimuli placed in the far location were not accessible by the participant. After each hand action participants were required to return their hand back to the starting position. Object locations were predetermined during the participant setup; participants placed the objects down at the near and medium locations as determined by the extent of their reach, with the far location being placed by the experimenter at the far edge of the platform outside the reach of the participant. Pieces of black tape were positioned at the three locations to aid the experimenter in placing the stimuli in the correct locations in the dark. Small LEDs were positioned underneath the platform and directed toward the experimenter such that they were not visible to the participant but could cue the experimenter regarding the location of the upcoming target to be placed on the platform during the intertrial interval. During participant setup, the illuminator LED was positioned to provide equal illumination to stimuli in the three prespecified object locations. All stimuli were positioned below the fixation point, in the lower visual field. Participants were instructed to ignore objects that were fumbled and to only make one attempt at a grasp or reach. The experimenter watched the participants' actions and noted any trials with errors; error trials were coded as predictors of no interest and excluded from analyses. A separate practice session was conducted before the actual experiment to familiarize participants with the paradigm. This practice session was equivalent to one experimental functional run.

Each functional run was comprised of 20 trials [four trials for each of the five conditions: grasp medium object, reach medium object, passive viewing of near object $\left(\mathrm{Near}_{\mathrm{pv}}\right)$, passive viewing of medium object (Me$\operatorname{dium}_{\mathrm{pv}}$ ), passive viewing of far object $\left(\mathrm{Far}_{\mathrm{pv}}\right)$ ] (Fig. 1C). During each run, trials were pseudo-randomly ordered. All participants participated in five functional runs.

Data acquisition. Imaging was performed at the Robarts Research Institute (London, Ontario, Canada) using a 4 Tesla whole-body SiemensVarian MRI system. A single-channel, transmit-receive, cylindrical birdcage radiofrequency head coil was used in all experiments. BOLD-based (Ogawa et al., 1992) functional MRI volumes were collected using an optimized segmented $\mathrm{T} 2^{\star}$-weighted echoplanar imaging (EPI) acquisition. To optimize the signal:noise ratio, in experiment 1 we used multishot imaging in which k-space was collected using two shots per slice, each with a TR (repetition time) of $1000 \mathrm{~ms}$, for a total volume acquisition time of $2 \mathrm{~s}$ [flip angle (FA), $30^{\circ}$ ]. A $19.2 \mathrm{~cm}$ field of view was collected with a $64 \times 64$ matrix size, yielding an in-plane resolution of $3 \mathrm{~mm}$. Each volume comprised 15 contiguous oblique slices, each $6 \mathrm{~mm}$ thick. Slices were acquired at a $30^{\circ}$ caudal tilt with respect to the anterior commissure to posterior commissure (ACPC) line (Damasio, 1995), covering occipital, parietal, posterior temporal and posterior frontal cortices. A constrained, three-dimensional phase shimming procedure was performed to optimize the magnetic field homogeneity over the prescribed func- tional planes (Klassen and Menon, 2004). Physiologic fluctuations were compensated for every segment of every slice using a point-based navigator correction scheme collected at the beginning of every spiral read-out.

Data analysis. Data were analyzed using a Brain Voyager QX software package (Version 1.8.6, Brain Innovation). For each participant, functional data from each session were screened for motion and/or magnet artifacts with cine-loop animation. One run from one participant was removed from analyses due to extreme head motion $(>1 \mathrm{~mm}$ within a run). For the remaining data, Brain Voyager's motion correction was applied to align each functional volume for a given participant to the functional volume collected closest in time to the anatomical volume. In addition, data were preprocessed with linear tend removal and a high pass filter of 3 cycles/run. Functional data were superimposed on anatomical brain images, aligned on the ACPC line, and transformed into Talairach space (Talairach and Tournoux, 1988). For group analysis, functional data were spatially smoothed using a Gaussian kernel of $8 \mathrm{~mm}$ (full-width at half-maximum). Group data were then analyzed using a random effects (RFX) general linear model (GLM). For experiment 1, predictors were generated using rectangular wave functions (with a value of 1 for 1 volume $=2 \mathrm{~s}$ when the action was initiated at the onset of the intertrial interval and a value of 0 for the remainder of the trial) that were convolved with a hemodynamic response function (Boynton et al., 1996). The data were processed using a percentage signal change transformation.

\section{Experiment 2: investigation of preference for reachable space at controlled locations after tool use}

Experiment 1 showed that SPOC has higher activation for passively viewing objects within reaching distance (near and medium object locations). The initial suggestion of this finding is that SPOC encodes the potential to act upon objects within reach of the hand and our left-lateralized SPOC activation is consistent with a coding of reachable objects with respect to the available contralateral right arm. There are, however, other possible interpretations of this finding. In experiment 1 , participants only ever acted on (grasped or reached toward) stimuli located at the medium object location. The experiment was designed this way to minimize the number of conditions and thus maximize statistical power for this preliminary investigation. However, it is possible that locations associated with actions (medium location) can become primed for actions during viewing conditions. As such, the heightened activation for the medium location could be unrelated to object reachability and may instead reflect a confounding factor in our first study. Similarly, the limited number of conditions in our preliminary investigation make it possible that activation differences in SPOC were due to visual confounds such as retinal size (near and medium locations appear larger on the retina), retinal eccentricity (the near and medium locations were most eccentric in the visual field), or the depth of fixation (near and medium objects were within the depth of vergence). Previous results have shown that activation near SPOC can be modulated by the depth of fixation (Quinlan and Culham, 2007).

Based on these preliminary findings in experiment 1 the goals of experiment 2 were twofold. First, we needed to control for alternative explanations of our results. To address these other interpretations we: (1) had participants perform actions (reaching and grasping) on object locations that were not passively viewed on other trials; (2) used objects of two different physical sizes so that when positioned at either near and far distances they provided the same retinal image size; (3) positioned objects in both the left and right visual fields at equal depths providing the same retinal eccentricities and the same depth relative to the fixation point; and (4) increased the depth of fixation so that both reachable and unreachable objects were positioned nearer than the fixation point. The second goal of experiment 2 was to investigate whether the range of reachable space encoding in SPOC could be expanded when participants are given a tool that extends their reach. To investigate this possibility, experiment 2 interleaved two types of functional runs in which the participant either used the hand $(2 a)$ or a tool $(2 b)$ to perform reaching and grasping actions. The expectation was that on runs in which the hand was used, the preferred spatial range would include only the targets reachable 
with the hand; whereas, on runs in which a tool was used, the preferred spatial range would extend to include targets that were reachable with the tool but not the hand alone.

Participants. Fourteen right-handed participants ( 4 females; mean age, 26 years) were recruited from the University of Western Ontario (London, Ontario, Canada) for this experiment. Data from one additional participant were discarded due to motion artifacts. Participants met the same inclusion criteria and underwent the same consent and screening procedures as in experiment 1. Each scanning session consisted of one high resolution anatomical acquisition and a minimum of 11 functional runs.

Experiment $2 a$ and $2 b$. The experimental setup, apparatus, and tasks were similar to experiment 1 except where otherwise noted. During odd-numbered runs, which constituted experiment $2 \mathrm{a}$, participants used the hand to reach and grasp objects on some trials; on other trials they simply passively viewed objects at a variety of locations. For action trials participants were required to perform the grasping and reaching actions with their right hand to an object located along the arc of reachability, at the point corresponding to the participant's sagittal midline (hand location, $\mathrm{H}$ ). During passive viewing conditions, participants were instructed to attend to one of five other object locations: two near locations [near right $\left(\mathrm{NR}_{\mathrm{pv}}\right)$ and near left $\left(\mathrm{NL}_{\mathrm{pv}}\right)$ locations] positioned in the right and left visual fields, equally eccentric from fixation (such that only the $\mathrm{NR}_{\mathrm{pv}}$ location was within range of the participant's reach); two middle locations on the left and right [medium left $\left(\mathrm{ML}_{\mathrm{pv}}\right)$ and medium right $\left(\mathrm{MR}_{\mathrm{pv}}\right)$ locations], both positioned at a unreachable further distance; and a far location [far right $\left(\mathrm{FR}_{\mathrm{pv}}\right)$ location] that was positioned in the right visual field far beyond reach of the hand (Fig. 2A).

During even-numbered runs, which constituted experiment $2 \mathrm{~b}$, participants used a tool to reach and grasp objects in some trials; on other trials, they simply passively viewed objects at the same locations as experiment 2a while holding the tool in hand. On action trials participants performed grasping and reaching with a set of large, plastic tongs to objects at a tool (T) location (along the arc of reachability with the tool, located along the participant's sagittal midline but further than the $\mathrm{H}$ location). On passive viewing trials, participants kept the tool in hand at the starting position while objects were presented at one of the same locations as experiment $2 \mathrm{a}\left(\mathrm{NR}_{\mathrm{pv}}, \mathrm{NL}_{\mathrm{pv}}, \mathrm{MR}_{\mathrm{pv}}, \mathrm{ML}_{\mathrm{pv}}, \mathrm{FR}_{\mathrm{pv}}\right)$. In this case, the range of space reachable with the tool now encompassed passive viewing locations $\mathrm{NR}_{\mathrm{pv}}, \mathrm{NL}_{\mathrm{pv}}$, and $\mathrm{MR}_{\mathrm{pv}}$ (Fig. $2 \mathrm{~B}$ ). Although including hand and tool trials within the same run would have been better for direct statistical comparisons between them, this would have led to too many conditions per run and would have necessitated insertion and removal of the tool, increasing run duration and possibly leading to movement artifacts.

The exact locations of the passively viewed object locations $\left(\mathrm{NR}_{\mathrm{pv}}\right.$, $\mathrm{NL}_{\mathrm{pv}}, \mathrm{MR}_{\mathrm{pv}}, \mathrm{ML}_{\mathrm{pv}}, \mathrm{FR}_{\mathrm{pv}}$ ) were determined individually for each participant. $\mathrm{NR}_{\mathrm{pv}}$ was placed within reach by the participant with $\mathrm{NL}_{\mathrm{pv}}$ placed at an equally distant location (with respect to the participant) outside the participant's reach by the experimenter (thus an activation preference for $\mathrm{NR}_{\mathrm{pv}}$ over $\mathrm{NL}_{\mathrm{pv}}$ in SPOC could not be explained in terms of retinal eccentricity as $\mathrm{NL}_{\mathrm{pv}}$ was equally eccentric from fixation but outside the reach of the participant's arm). Location $\mathrm{MR}_{\mathrm{pv}}$ was placed within reach of the tool by the participant themselves and object location $\mathrm{ML}_{\mathrm{pv}}$ was positioned in an equally distant location outside reach of the tool by the experimenter. Object location $\mathrm{FR}_{\mathrm{pv}}$ was placed beyond reach of the tool in the right visual field at the maximal extent of the platform by the experimenter (note that we did not include a symmetric location in the left visual field [far left (FL)] because the predictions would have been the same as for the $\mathrm{FR}_{\mathrm{pv}}$ condition and we wanted to minimize the number of
B) Experiment 2b

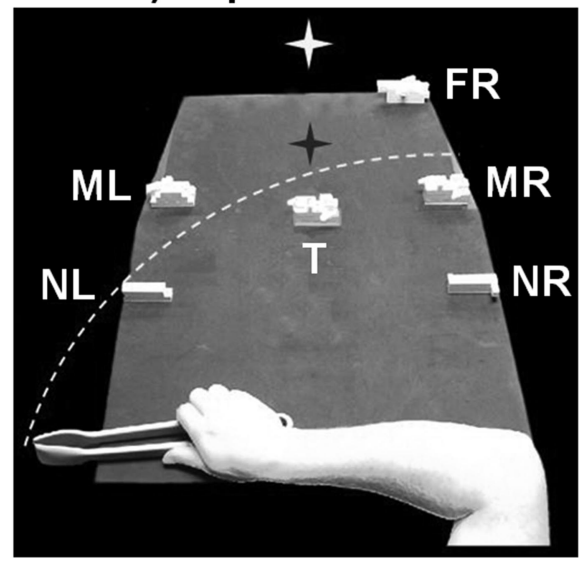

nt 2. On any given trial only one object would appear at any given

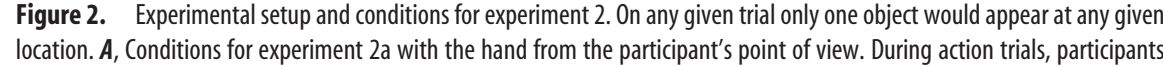
grasped and reached objects at location $\mathrm{H}$ with their hand while on passive viewing trials participants viewed an object at one of five locations: NR (near right), NL (near left), MR (middle right), ML (middle left), and FR (far right). $\boldsymbol{B}$, Conditions for experiment $2 \mathrm{~b}$ with the tool from the participant's point of view. Participants grasped and reached to objects at location T using the tool during represents the fixation LED and its location in depth (no shadow was actually visible). The white dashed line represents the extent of the participant's reaching space within the setup. Note that objects in medium and far locations are physically larger in size to provide a retinal size equivalent to that of the near objects.

trial types to maintain a reasonable number of trials per condition). These five passive viewing conditions included stimuli in both visual fields $\left(\mathrm{NR}_{\mathrm{pv}}, \mathrm{MR}_{\mathrm{pv}}\right.$ and $\mathrm{FR}_{\mathrm{pv}}$ on right, $\mathrm{NL}_{\mathrm{pv}}$ and $\mathrm{ML}_{\mathrm{pv}}$ on left). Thus, for experiment $2 \mathrm{a}$, if SPOC shows selectivity for the only reachable object location with the hand, $\mathrm{NR}_{\mathrm{pv}}$, and not other locations on the right $\left(\mathrm{MR}_{\mathrm{pv}}\right.$ and $\mathrm{FR}_{\mathrm{pv}}$ ), we can rule out that SPOC activation is influenced by an object's visual field location. Also note that participants performed grasping and reaching with the hand (2a) and the tool (2b) to object locations corresponding to the participant's midline ( $\mathrm{H}$ and $\mathrm{T}$ locations, respectively) and importantly, objects were not presented at these locations during any passive viewing trials. This manipulation controlled for two factors: First, these actions locations were precisely along the midline such that participants were not biased to act toward either the left or right side of space. Second, by separating passive viewing locations from action locations, heightened activation at any one passive viewing location will be independent of any previous motor associations at that location.

To control for object retinal size in experiment 2, two LEGO object sizes, large and small, were created so that objects at different depth locations provided the same retinal image size for the participant. Large object stimuli $[7 \mathrm{~cm}$ (length) $\times 3 \mathrm{~cm}$ (depth) $\times 3 \mathrm{~cm}$ (height) $]$ were placed at locations $\mathrm{ML}_{\mathrm{pv}}, \mathrm{MR}_{\mathrm{pv}}, \mathrm{FR}_{\mathrm{pv}}$, and $\mathrm{T}$ and small object stimuli $(5 \times 1.5 \times 2 \mathrm{~cm})$ were placed at locations $\mathrm{NR}_{\mathrm{pv}}, \mathrm{NL}_{\mathrm{pv}}$, and $\mathrm{H}$. With these physical sizes, the large stimuli at the medium distance subtended a visual angle of $4 \times 1.7 \times 1.7$ degrees in length, depth and height. The small stimuli at the near distance subtended $4 \times 1.2 \times 1.6$ degrees of visual angle. Although the sizes of available LEGO pieces did not allow exact equivalence, the near stimuli were now retinally smaller than the far stimuli, reversing the size difference from experiment 1 . Thus, a replication of the results from experiment 1 would rule out retinal size as the basis for activation differences in SPOC. The fixation point was placed at a depth between the medium and far object locations (similar to experiment 1); however, note that this fixation distance was further from the participant than in our first experiment and all stimuli fell within the lower visual field. There were three other differences from experiment 1 : (1) LEGO stimuli were painted white to increase their contrast with the black background of the platform; (2) The platform was extended to enable the placement of objects at further distances beyond range of the tool; and (3) the timing was changed so that each event-related trial lasted $15 \mathrm{~s}$ (based upon a volume acquisition time of $1.5 \mathrm{~s}$ for 10 volumes per 


\section{Single Subject Results}

\section{Group Voxelwise Results}
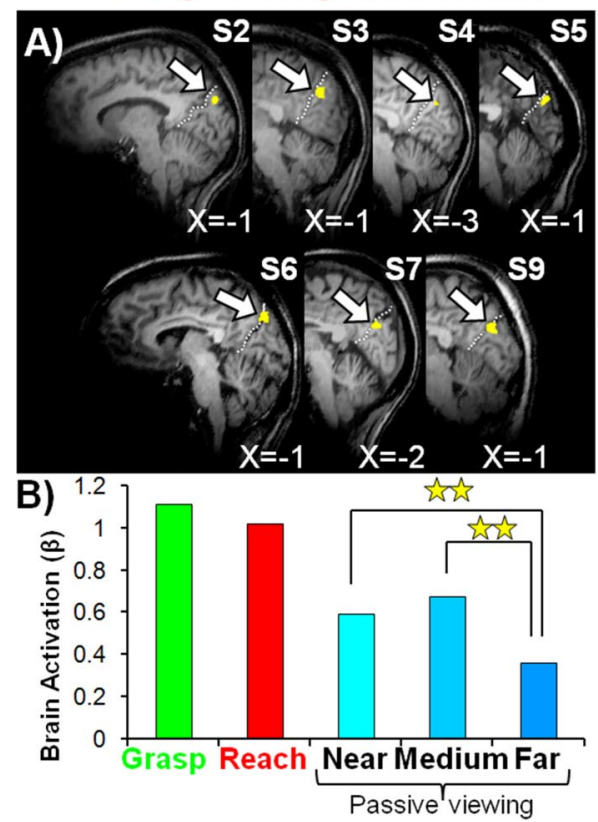
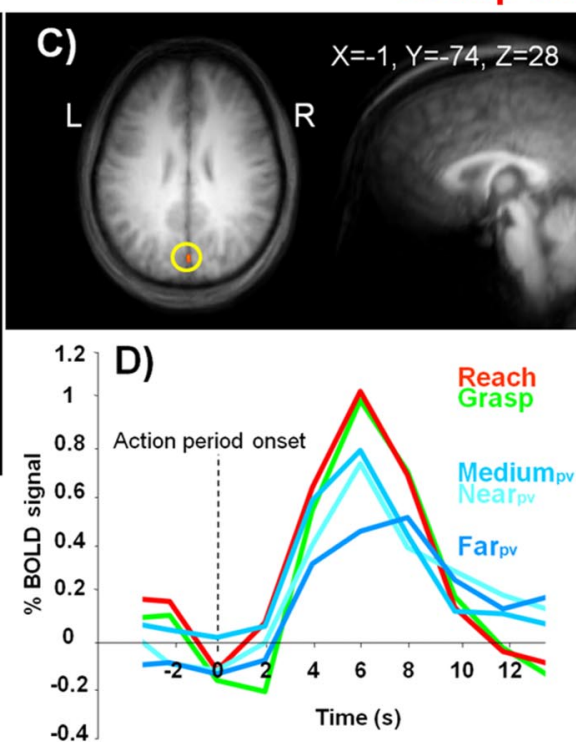

E)

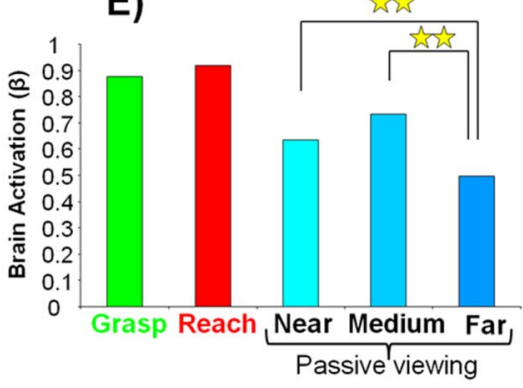

$\widehat{\imath} 2$ denotes significance at $a<0.01$

Figure 3. Single-subject and group voxelwise results for experiment 1. $A$, Overlay of fMRI region-of-interest activation for the passive viewing of reachable versus unreachable objects [(Near ${ }_{\mathrm{pv}}$

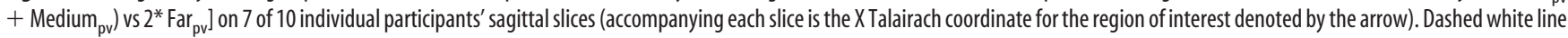
corresponds to the parieto-occipital sulcus. $\boldsymbol{B}$, Averaged $\beta$ weights from the selected areas in $\boldsymbol{A}$. $\boldsymbol{C}$, Overlay of fMRI activation for reachable versus unreachable objects [(Near ${ }_{\mathrm{pv}} \mathrm{vs}^{\mathrm{F}} \mathrm{Far}_{\mathrm{pv}}$ ) and $\left(\right.$ Medium $_{\mathrm{pv}} \mathrm{vs} \mathrm{Far}_{\mathrm{pv}}$ )] on a group averaged anatomical (using RFX analysis). Region defined by yellow circle denotes SPOC. D, \% BOLD signal change from the selected region in C. $\boldsymbol{E}, \beta$ Weight activation from the selected region in $\boldsymbol{C}$.

trial) instead of the $16 \mathrm{~s}$ in experiment 1 . This latter change only affected the length of the preparation period, which was shortened to $5 \mathrm{~s}$. As in experiment 1 , participants were instructed to always maintain fixation on a green LED during the entire length of a run.

Task. As in the first experiment, in experiment 2, participants were asked to respond to the cued auditory instruction ("grasp," "reach," or "look") as accurately as possible during the $2 \mathrm{~s}$ while the stimuli were illuminated by an LED. Grasping with the tool involved using the ends of the tongs (driven by forces of the thumb and index finger to the middle of the tongs) to acquire, briefly lift, and replace the object. The reaching condition with the tool required that the participant reach toward the object, close the ends of the tongs, and touch the object with the tongs. Participants were instructed to keep the actions and somatosensory feedback as similar as possible for grasping and reaching trials, for example, by exerting similar pressure on the sides of the tongs and using similar timing. Error trials were viewed off-line using an MRcompatible infrared-sensitive camera (which had not been available for experiment 1) that was optimally positioned to record the participant's movements during functional runs (MRC Systems). Trials containing errors were modeled as predictors of no interest and were excluded from statistical contrasts.

A separate practice session was conducted before the actual experiment to familiarize participants with the tool and the paradigm. This practice session was performed during collection of the participant's anatomical scan and was both similar to a tool functional run and equivalent to the length of one experimental functional run. Participants were also given brief practice with the tool picking up objects at several different locations on the platform before the anatomical scan. Each hand (2a) and tool (2b) functional run was comprised of 21 trials, based on a quasi-random ordering of three trials for each of the seven conditions: grasp with hand/tool (dependent on run), reach with hand/tool (dependent on run), and passive viewing an object at one of five locations $\mathrm{NR}_{\mathrm{pv}}$, $\mathrm{NL}_{\mathrm{pv}}, \mathrm{MR}_{\mathrm{pv}}, \mathrm{ML}_{\mathrm{pv}}$, or $\mathrm{FR}_{\mathrm{pv}}$.

Data analysis for experiment 2 . All data analyses were the same as in experiment 1 except where otherwise noted. All data from one participant and one run from each of two additional participants were removed from analyses due to extreme head motion ( $>1 \mathrm{~mm}$ within a run). A timing adjustment in experiment 2 led to a slight difference in the duration of the rectangular wave functions (that now had a value of 1 for 1 volume $=1.5 \mathrm{~s}$ when the action was initiated at the onset of the intertrial interval and a value of 0 for the remainder of the trial), which were then convolved with a two-gamma hemodynamic response function (Brain Voyager QX). Data for group voxelwise analyses (but not single-subject analyses) were spatially smoothed using a Gaussian kernel of $8 \mathrm{~mm}$ (fullwidth at half-maximum). Data were preprocessed with a linear trend removal and a high pass filter of 2 cycles/run. To allow for the possibility of comparing between trial types in the hand and tool runs, the general linear model included 14 predictors, one for each trial type (hand vs tool $\times 7$ conditions). In hand runs, the predictors for tool trials were flat, while in tool runs, the predictors for hand trials were flat. Comparisons between the hand and tool runs assume that the levels of activation during the baseline period were comparable, which is reasonable because the baseline tasks were identical. The interleaving of hand and tool runs makes it unlikely that any differences between the two conditions were due to systematic changes such as fatigue.

\section{Results}

Experiment 1

Single-subject analysis

An area tuned to reachable space would be expected to show higher visual responses for the viewing of objects within reach $\left(\right.$ Near $_{\mathrm{pv}}$ and Medium $\left.\mathrm{pv}_{\mathrm{v}}\right)$ versus objects outside of reach $\left(\mathrm{Far}_{\mathrm{pv}}\right)$. Thus, within each of our 10 subjects we contrasted activation for the passive viewing of reachable objects vs the unreachable object locations $\left[\left(\mathrm{Near}_{\mathrm{pv}}+\right.\right.$ Medium $\left._{\mathrm{pv}}\right) \mathrm{vs} 2^{\star} \mathrm{Far}_{\mathrm{pv}}$ ]. This contrast identified clusters of activation in seven of 10 subjects (averaged Talairach coordinates: $X=-1, Y=-76, Z=26$ ) in SPOC at the superior end of the parieto-occipital sulcus (POS) (Fig. 3A), which separates the occipital and parietal cortices on the medial surface. This activity was analyzed in individuals by capturing all the activation at a threshold of $p<0.01$ within a cube of $(10$ $\mathrm{mm})^{3}$ centered on the single most statistically significant voxel 
within SPOC. The contrast used to select the SPOC region of interest (ROI) necessitated that the averaged $\beta$ weights across these subjects show a preference for reachable versus nonreachable objects (Fig. 3B), and post hoc comparisons confirmed that

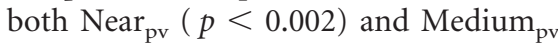
$(p<0.01)$ were significantly greater than

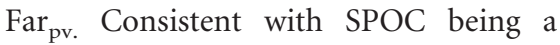
reach-related region (Prado et al., 2005; Cavina-Pratesi et al., 2006a) we also observed that grasping and reaching actions were significantly higher than all the passive viewing conditions (minimum of $p<$ 0.05 ). Also of interest (but not shown in Fig. $3 A$ ), in five of these subjects we observed single foci of activation situated medially in the occipital lobe above the calcarine sulcus, likely corresponding to the presentation of visual stimuli within the lower visual field during passive viewing trials.

\section{Group analysis}

To confirm our single-subject findings we then used an RFX GLM to perform a conjunction analysis for the contrasts of $\left(\right.$ Near $_{\mathrm{pv}}>$ Far $\left._{\mathrm{pv}}\right)$ and $\left(\right.$ Medium $_{\mathrm{pv}}>$ Far $\left._{\mathrm{pv}}\right)$. At the group level, we were able to employ this more conservative conjunction analysis instead of the less strict contrast of $\left[\left(\mathrm{Near}_{\mathrm{pv}}+\right.\right.$ Medium $\left._{\mathrm{pv}}\right)$ vs $2^{*}$ $\left.\operatorname{Far}_{\mathrm{pv}}\right]$. This contrast identified SPOC activation, as shown in Figure $3 C(t=3.2, p<$ $0.01,32 \mathrm{~mm}^{3}$; Talairach coordinates: $X=$ $-1, Y=-74, Z=28)$. Again, consistent with the contrast used to select the cluster, its time course and $\beta$ weight activations showed activity dependent on the reachability of the object locations (Fig. 3D,E). For a conjunction analysis the effective $p$ value is the square of the $p$ values for each component (here $0.01^{2}=0.0001$ ), which is a respectable threshold for random effects analysis with only 10 participants (the minimum recommended sample size for RFX). As presented later, we were able to replicate this result in experiment 2 with the appropriate cluster size correction when the sample size was larger $(n=14)$.

Not surprisingly based on this contrast used to identify SPOC, we observed greater activation for both the $\operatorname{Near}_{\mathrm{pv}}(p<0.007)$ and Medium ${ }_{\mathrm{pv}}$ conditions $(p<0.001)$ compared with the Far $_{\mathrm{pv}}$ condition. It is unlikely that these findings in left SPOC can be attributed to a retinotopic response given that this area shows a preference for both ipsilateral $\left(\mathrm{Near}_{\mathrm{pv}}\right)$ and contralateral (Medi$\mathrm{um}_{\mathrm{pv}}$ ) visual field locations. We also found higher responses for reaching (minimum of $p<0.01$ ) and reach-to-grasp (minimum of $p<0.05$ ) actions compared with the passive viewing responses (with the exception that grasping and reaching were not significantly greater than Medium $_{\mathrm{pv}}$ ). With the above conjunction analysis we also observed a small area of activation situated medially in the occipital lobe above the calcarine sulcus (Talairach coordinates: $X=-1, Y=-77, Z=10$ ) (Fig. 3C), similar to that observed in single subjects.

\section{Single Subject Results}

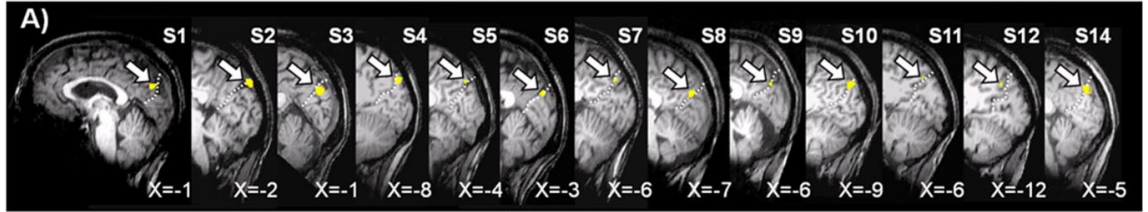

B)

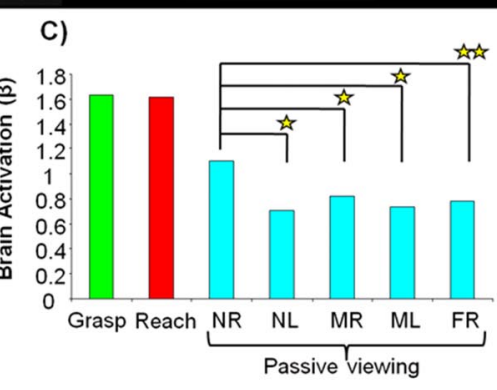

HAND

TOOL

는 denotes significance at $a<0.01$ $\sum$ denotes significance at $a<0.05$

\section{Group Voxelwise Results}
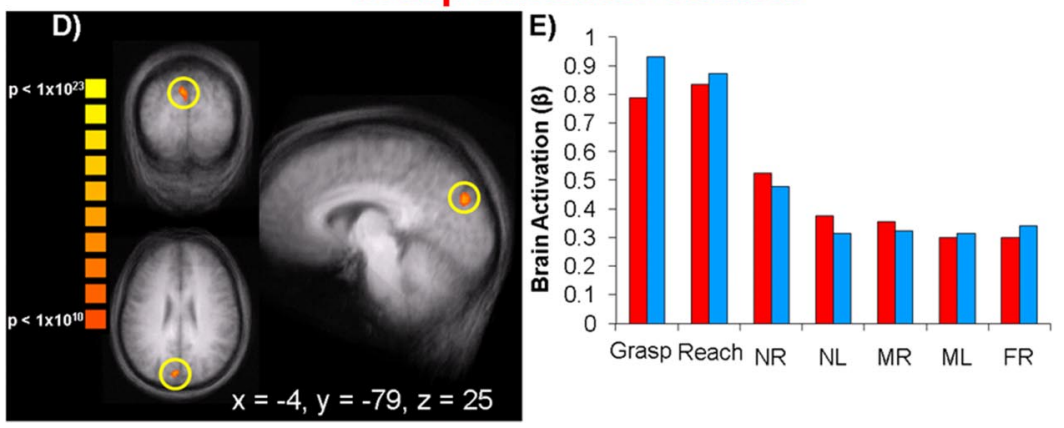

- hand $\square$ tool

Figure 4. Single-subject and group voxelwise results for experiment 2. A, Overlay of fMRI region-of-interest activation for the passive viewing of reachable versus unreachable objects with respect to the hand $\left[4^{*} \mathrm{NR}_{\mathrm{pv}} \mathrm{vs}\left(\mathrm{NL}_{\mathrm{pv}}+\mathrm{MR}_{\mathrm{pv}}+\mathrm{ML}_{\mathrm{pv}}+\mathrm{FR}_{\mathrm{pv}}\right)\right]$ on 13 of 14 individual participants' sagittal slices (accompanying each slice is the X Talairach coordinate for the region of interes areas in $\boldsymbol{A}$ for functional runs with the hand. $\boldsymbol{C}$, Averaged $\beta$ weights from selected areas in $\boldsymbol{A}$ for functional runs with the tool. $\boldsymbol{D}$, Overlay of fMRI activation for reachable versus unreachable objects $\left[4^{*} \mathrm{NR}_{\mathrm{pv}} \mathrm{vs}\left(\mathrm{NL}_{\mathrm{pv}}+\mathrm{MR}_{\mathrm{pv}}+\mathrm{ML}_{\mathrm{pv}}+\mathrm{FR}_{\mathrm{pv}}\right)\right]$ on a group averaged anatomical (using FFX analysis, cluster threshold corrected, $380 \mathrm{~mm}^{3}$ ). Region defined by yellow circle denotes SPOC. Accompanying the region of interest are its Talairach coordinates. $\boldsymbol{E}, \beta$ Weight activation from the selected region in $\boldsymbol{D}$ for statistically higher than each of the nonreachable object locations ( $\mathrm{NL}_{\mathrm{pv}}, \mathrm{MR}_{\mathrm{pv}}, \mathrm{ML}_{\mathrm{pv}}$, and $\mathrm{FR}_{\mathrm{pv}}$ ). NR, Near right; $\mathrm{NL}$, near left; $M R$, middle right; $M L$, middle left; $F R$, far right.

\section{Experiment 2}

Single-subject analysis in hand runs (experiment 2a)

To localize SPOC in a similar way as in experiment 1 , we first used the data from functional runs with the hand (experiment $2 a$ ) for each of the 14 participants. We contrasted activation for the passive viewing of the reachable object location versus the unreachable objects locations $\left[4^{\star} \mathrm{NR}_{\mathrm{pv}}\right.$ vs $\left(\mathrm{NL}_{\mathrm{pv}}+\mathrm{MR}_{\mathrm{pv}}+\mathrm{ML}_{\mathrm{pv}}+\right.$ $\mathrm{FR}_{\mathrm{pv}}$ )] [note that we did not use a conjunction analysis contrast of $\mathrm{NR}_{\mathrm{pv}}$ vs each of the nonreachable passive viewing conditions ( similar to experiment 1 group analysis) because it did not identify SPOC or any other brain areas at an acceptable statistical threshold, likely because corrections for multiple comparisons make it difficult for an area to simultaneously satisfy all four contrasts]. This contrast revealed activations in SPOC in 13 of 14 participants (Fig. $4 \mathrm{~A}$ ), very close to the focus of activation found in experiment 1 at the superior end of the POS. Although the activation in individuals was sometimes anterior to the POS and sometimes posterior to it, it was always consistently at the superior end of the POS (averaged Talairach coordinates: $X=-5$, 


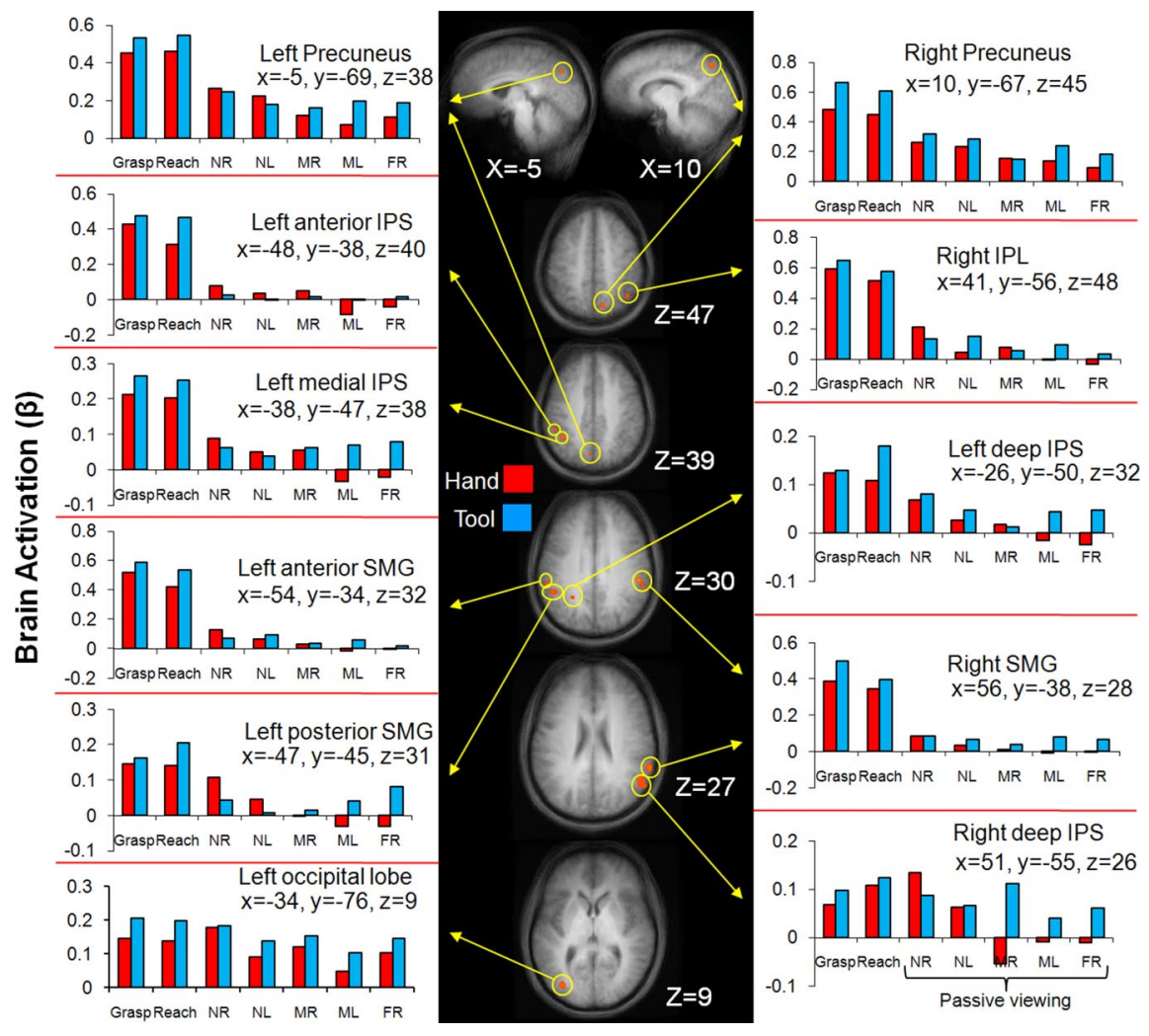

Figure 5. Areas activated by a group voxelwise contrast for the passive viewing of reachable versus unreachable objects with respect to the hand $\left[\mathrm{NR}_{\mathrm{pv}} \mathrm{vs}\left(\mathrm{NL}_{\mathrm{pv}}+\mathrm{MR}_{\mathrm{pv}}+\mathrm{ML}_{\mathrm{pv}}+\mathrm{FR}_{\mathrm{pv}}\right)\right]$ overlayed on group averaged anatomical slices (using RFX analysis). Located near each anatomical slice is its relevant Talairach coordinate. $\mathrm{fMRI}$ activation is shown in orange at $p<0.001$ with its accompanying $\beta$ weight activation denoted by yellow arrows. Note that the scale of brain activation changes across brain areas. $\mathrm{NR}$, Near right; $\mathrm{NL}$, near left; $M R$, middle right; $M L$, middle left; $F R$, far right.

$Y=-78, Z=28$ ). This activity was gathered from individuals by capturing all the activation at a threshold of $p<0.01$ within a cube of $(10 \mathrm{~mm})^{3}$ centered on the single most statistically significant voxel within SPOC. Although the contrast used to identify this region specified only that the $\mathrm{NR}_{\mathrm{pv}}$ location had to yield higher activation than the average of the other four locations, statistical contrasts performed on the averaged $\beta$ weights in SPOC found significant differences between the $\mathrm{NR}_{\mathrm{pv}}$ location compared with each of the unreachable passive viewing locations $\left(\mathrm{NR}_{\mathrm{pv}}\right.$ vs $\mathrm{NL}_{\mathrm{pv}}, p<0.003 ; \mathrm{NR}_{\mathrm{pv}}$ vs $\mathrm{MR}_{\mathrm{pv}}, p<0.003 ; \mathrm{NR}_{\mathrm{pv}}$ vs $\mathrm{ML}_{\mathrm{pv}}, p<0.002 ; \mathrm{NR}_{\mathrm{pv}}$ vs $\left.\mathrm{FR}_{\mathrm{pv}}, p<0.005\right)$ with no difference among unreachable locations (Fig. $4 B$ ). These findings independently confirmed the results of the first experiment, reinforcing the conclusion that SPOC encodes objects reachable by the hand. Post hoc comparisons on the $\beta$ weights in SPOC also showed that grasping and reaching activation were not statistically different from one another; however, both conditions were higher than the passive viewing conditions.

\section{Group analysis in hand runs (experiment 2a)}

In addition to the single-subject analyses, we also performed voxelwise RFX analyses on the group data averaged in stereotaxic space. Using the same contrast used to identify SPOC in single subjects $\left[4^{*} \mathrm{NR}_{\mathrm{pv}}\right.$ vs $\left(\mathrm{NL}_{\mathrm{pv}}+\mathrm{MR}_{\mathrm{pv}}+\mathrm{ML}_{\mathrm{pv}}+\mathrm{FR}_{\mathrm{pv}}\right)$, we found several foci of activation (Fig. 5), including bilateral activation in the precuneus, $\sim 1 \mathrm{~cm}$ anterior to the superior parieto-occipital location where SPOC would be expected. In post hoc comparisons, both left and right precuneus showed higher activation for the $\mathrm{NR}_{\mathrm{pv}}$ location versus the two medium and far locations (all $p$ values $<0.003$ ), but no significant difference in activation between $\mathrm{NR}_{\mathrm{pv}}$ and $\mathrm{NL}_{\mathrm{pv}}$ (and $\mathrm{NL}_{\mathrm{pv}}$ did not differ significantly from any of the other locations except $\mathrm{NL}_{\mathrm{pv}}$ versus $\mathrm{FR}_{\mathrm{pv}}$ in the right precuneus, $p<$ 0.029). It is worth noting, however, that in all the other nine areas identified (Fig. 5), the signals were weak and not particularly reliable. For all of these areas, the activation for passive viewing conditions was $<0.2 \%$ signal change (according to the percentage-transformed $\beta$ weights), considerably weaker than the percentage signal change in left SPOC (between 0.6\% and $1.4 \%$ ) (Fig. 4). Moreover, only four of these areas, the left posterior supramarginal gyrus (SMG), the right SMG, the right inferior parietal lobule (IPL) and the left occipital lobe, met the requirement that $\mathrm{NR}_{\mathrm{pv}}$ be significantly higher than each of the other unreachable passive viewing locations in post hoc contrasts. In addition, unlike left SPOC in the single-subject analysis and fixed effects (FFX) group analyses, the passive viewing activation patterns were not replicated in the tool runs.

Based on the robust SPOC activations from both experiment 1 and single-subject analyses we were initially surprised that our RFX contrast did not reveal SPOC at the group level. Note, however, that although RFX analyses have become the norm because they show intersubject consistency, they may fail to find common activation despite intersubject consistency simply because of the variability in stereotaxic coordinates across subjects. Indeed, when we performed a less stringent FFX analysis on the data (Fig. 4D), we observed SPOC activation. Certainly our single-subject analyses showed (as RFX is typically intended to do) that SPOC shows a preference for reachable space in virtually all participants. Thus, although our SPOC activation was consistent across individuals with respect to the POS (Fig. 4A), the POS is quite stereotaxically variable across subjects making it difficult for individual voxels in SPOC to have enough consistency to pass RFX criteria. Other differences between the areas activated in the two experiments may be related to differences in design and analyses including (1) the number of trial types and the contrasts used and (2) differences in the number of trials ( $\sim 15$ trials vs $\sim 20$ trials in experiment 1$)$ and statistical power.

The findings of experiment $2 \mathrm{a}$, which eliminated possible confounds of our first study, strongly corroborate our initial finding that SPOC encodes object reachability with respect to the hand. In particular, in experiment $2 \mathrm{a}$ we showed that SPOC does not encode retinal size by incorporating two physical object sizes so that objects at different distances provide very similar retinal image sizes. We also ruled out the possibility that SPOC activation simply reflects retinal eccentricity by pairing object locations with equidistant, symmetric locations (for example $\mathrm{NR}_{\mathrm{pv}}$ and $\mathrm{NL}_{\mathrm{pv}}$ ). It is also unlikely that SPOC encodes all object locations nearer than the fixation point because similar results were obtained when the fixation point was placed at two different physical distances in experiments 1 and 2. Similarly, SPOC does not encode visual field because it shows no preferences for locations $\mathrm{MR}_{\mathrm{pv}}$ and $\mathrm{FR}_{\mathrm{pv}}$, 
which, like location $\mathrm{NR}_{\mathrm{pv}}$, are also located within the right visual field. Experiment 2 also showed that the visual responses in SPOC are independent from previous motor associations at certain object locations. Recall that in experiment 1 participants acted on the same object location they passively viewed (medium location); however, in experiment 2, we separated the acted and passively viewed object locations.

We did not monitor eye movements in either study simply because the setup time was already considerable and the addition of eye tracker positioning and calibration would have made the fMRI session unduly long and uncomfortable for the participants. Nevertheless, we do not believe that eye movement confounds are likely to account for our results. First, although eye movement confounds are a frequent source of concern, in all cases where our lab has monitored eye movements, we found that participants can indeed accurately maintain fixation as instructed (Quinlan and Culham, 2007), even in tasks that are much more demanding than our passive viewing task (Culham et al., 1998; Verstraten et al., 2001). Second, although we have previously shown that SPOC is modulated by vergence distance (Quinlan and Culham, 2007), if participants had looked directly at the targets, we should have observed comparable activation levels for both targets in near space in experiment $2\left(\mathrm{NR}_{\mathrm{pv}}\right.$ and $\left.\mathrm{NL}_{\mathrm{pv}}\right)$ rather than higher activation for the reachable target $\left(\mathrm{NR}_{\mathrm{pv}}\right)$ than the unreachable one $\left(\mathrm{NL}_{\mathrm{pv}}\right)$. Third, as we showed in experiment 2, SPOC activation was remarkably consistent across all 13/14 subjects, including those who were highly experienced fMRI participants used to maintaining fixation and those who were less experienced.

\section{SPOC activation in tool runs (experiment $2 b$ )}

We extracted the $\beta$ weights from functional runs with the tool (experiment $2 \mathrm{~b}$ ) for the SPOC region localized in single participants by the contrast of reachable versus nonreachable locations with the hand (experiment 2a). As shown in Figure 4C, for the passive viewing conditions, there was again heightened activation for the $\mathrm{NR}_{\mathrm{pv}}$ location compared with each of the other passive viewing locations $\left(\mathrm{NL}_{\mathrm{pv}}, p<0.03\right.$; $\mathrm{MR}_{\mathrm{pv}}, p<0.02 \mathrm{ML}_{\mathrm{pv}}, p<$ $\left.0.02 ; \mathrm{FR}_{\mathrm{pv}}, p<0.002\right)$, with no significant differences among $\mathrm{NL}_{\mathrm{pv}}, \mathrm{MR}_{\mathrm{pv}}, \mathrm{ML}_{\mathrm{pv}}$, and $\mathrm{FR}_{\mathrm{pv}}$ (Fig. $4 C$ ). Again, grasping and reaching with the tool were not statistically different from each other but were higher than the passive viewing conditions. The finding that SPOC shows a higher response to the passive viewing of stimuli in the $\mathrm{NR}_{\mathrm{pv}}$ location, reachable with the hand, is contrary to our prediction that with tool use the preferred range within SPOC would extend to object locations $\mathrm{NR}_{\mathrm{pv}}, \mathrm{NL}_{\mathrm{pv}}$, and $\mathrm{MR}_{\mathrm{pv}}$. One interpretation of this finding may be that subjects did not receive adequate practice with the tool to see the predicted effects; however, further investigation has shown that SPOC activation for tool runs is not modulated by the amount of practice with a tool across the length of a testing session. Further discussion of the absence of modulation by the tool is presented in the supplemental materials, available at www.jneurosci.org.

We also examined other areas of the brain that might be responsive to tool use. We questioned whether area aIPS, known to respond more to grasping than reaching with the hand (Culham et al., 2003), would also display activation greater for grasping when subjects acted using a tool. We show evidence (see supplemental material, available at www.jneurosci.org) that aIPS selectively encodes grasping actions independent of the effector used (hand or tool). This finding supports the results from several methodologies (neuroimaging, neurophysiology, and kinematics) suggesting that some action-related areas of the brain encode the goals of actions, rather than movements (Gentilucci et al., 2004; Hamilton and Grafton, 2006; Umilta et al., 2008).

Numerous additional exploratory contrasts did not reveal any significant activation $(p<0.01)$. These included contrasts such as: (1) passive viewing of reachable versus unreachable locations with the tool: $\left[2^{*}\left(\mathrm{NR}_{\mathrm{pv}}+\mathrm{NL}_{\mathrm{pv}}+\mathrm{MR}_{\mathrm{pv}}\right)-3^{*}\left(\mathrm{ML}_{\mathrm{pv}}+\mathrm{FR}_{\mathrm{pv}}\right)\right]$ in tool runs; (2) passive viewing of locations that became reachable with the tool (in the tool runs) versus those same locations without a tool (in the hand runs): $\left[\left(\mathrm{MR}_{\mathrm{pv}}+\mathrm{NL}_{\mathrm{pv}}\right)\right.$ in tool runs $\left(\mathrm{MR}_{\mathrm{pv}}+\mathrm{NL}_{\mathrm{pv}}\right)$ in hand runs]; (3) unreachable versus reachable locations with the hand: $\left[\left(\mathrm{NL}_{\mathrm{pv}}+\mathrm{MR}_{\mathrm{pv}}+\mathrm{ML}_{\mathrm{pv}}+\mathrm{FR}_{\mathrm{pv}}\right)-\right.$ $\left.4^{*}\left(\mathrm{NR}_{\mathrm{pv}}\right)\right]$ in hand runs; and (4) far versus near locations regardless of reachability: $\left[\left(\mathrm{MR}_{\mathrm{pv}}+\mathrm{ML}_{\mathrm{pv}}\right)-\left(\mathrm{NR}_{\mathrm{pv}}+\mathrm{NL}_{\mathrm{pv}}\right)\right]$ in hand runs.

We do not believe that the activation we observed in left SPOC in both experiments simply reflects a retinotopic representation of the lower right visual field. In particular, this explanation cannot account for experiment 1 findings of higher activation for targets in both the lower right and lower left visual fields. Nevertheless, to provide an additional test of this alternate explanation, we examined whether right SPOC activation could be observed for the near left targets (vs the other target locations). That is, if the activation we observed in left SPOC for the near right location was merely a retinotopic response, we should observe similar activation in right SPOC for the near left location. Thus, we contrasted the nearest left object location versus all other passive viewing locations for both experiments. In experiment 1 , we identified left SPOC using single-subject ROIs; thus we now evaluated whether right SPOC would be activated in single subjects with the complementary contrast used to find left SPOC: $\left[2^{*}\right.$ Near $_{\mathrm{pv}}$ vs $\left(\right.$ Medium $\left._{\mathrm{pv}}+\mathrm{Far}_{\mathrm{pv}}\right]$. This contrast, at the same threshold as that used to find left SPOC in seven subjects $(p<$ $0.01)$, revealed activation in only one individual in the vicinity of right SPOC. In experiment 2, left SPOC was also identified in single-subject ROIs so we also examined whether right SPOC was revealed in individual participants by the complementary contrast at the same threshold used to find left SPOC: $\left[4^{\star} \mathrm{NL}_{\mathrm{pv}}\right.$ vs $\left(\mathrm{NR}_{\mathrm{pv}}+\mathrm{MR}_{\mathrm{pv}}+\mathrm{ML}_{\mathrm{pv}}+\mathrm{FR}_{\mathrm{pv}}\right)$ with hands runs at $\left.p<0.01\right]$. This analysis showed activation in the vicinity of right SPOC in only 3 of 14 subjects, a much smaller proportion than the proportion, 13 of 14 subjects, which demonstrated left SPOC with its respective contrast. Given that right SPOC activation was weak or absent in both these analyses, the visual encoding in SPOC is unlikely to reflect a retinotopic response and instead appears strongly coupled to reachability.

\section{Discussion}

In two separate imaging experiments, we demonstrated that SPOC was more active for objects within reach than objects beyond reach even when no action was required. The focus of activation was highly consistent across experiments despite methodological differences in the locations tested.

\section{Role of SPOC in reaching and potential reaching}

A growing body of evidence indicates that human SPOC is involved in the guidance of arm movements (de Jong et al., 2001; Astafiev et al., 2003; Connolly et al., 2003; Prado et al., 2005; Cavina-Pratesi et al., 2006b; Pellijeff et al., 2006). More compellingly, lesions to the area are associated with the severe reaching deficits observed in optic ataxia (Karnath and Perenin, 2005). Our analyses suggest that the robust visual responses to reachable objects in SPOC reflect the potential of acting on them. These results are consistent with neurophysiological evidence for the 
coding of intention and target selection in parietal cortex (Snyder et al., 1997, 2000; Scherberger and Andersen, 2007). Moreover, they further suggest that objects may automatically evoke affordances, that is, potential actions to be performed on certain objects (Gibson, 1979), even if such actions are not explicitly planned. Consistent with this interpretation, the reach-related SPOC may inherently differentiate between objects affording potential actions (unreachable objects that can become reachable via a change in posture) versus objects affording immediate actions (objects directly reachable by the arm) for reach planning purposes. In accordance with this view, some patients with frontal cortical damage, which receives heavy projections from superior parietal cortex (Matelli et al., 1998; Shipp et al., 1998) in macaques, are unable to inhibit actions to graspable objects placed within reaching distance, in a disorder called alien hand syndrome (L'Hermitte, 1983; Della Sala et al., 1991; Chan and Ross, 1997).

Given that all participants who participated in this study were right-handed and only ever acted with their right arm, it is not surprising that our activation in SPOC is lateralized to the left hemisphere. Participants were required to position their left arm close to their body (constrained underneath the platform) so as not to obstruct their viewpoint or interfere with actions of the right arm. Interestingly, contralateral SPOC activation suggests that either the area was sensitive to the constraints upon the left hand, or shows responses related only to reachability with the dominant right hand (Handy et al., 2003).

Our study suggests that SPOC does not encode near space exclusive of reachability. This possibility is certainly one to consider, particularly given another experiment from our lab which showed that SPOC responses increased as gaze converged (Quinlan and Culham, 2007). Here we have shown that even when gaze was fixed at a constant depth, the response in SPOC during passive viewing depended on whether objects were within reachable space. Unfortunately, because no common subjects were used, direct comparisons between projects were not possible. Although speculative, we propose that SPOC may use signals about gaze depth, combined with information about object depth with respect to gaze (e.g., from binocular disparity) to determine whether or not the object is within reach.

We chose not to include an attentional task in the passive viewing conditions because it may have distorted the nature of processing within parietal cortex; nevertheless, we do not believe that attentional confounds can account for the observed results. First, participants were kept alert by the need to perform grasping and reaching actions on some trials. Second, SPOC was the only area activated across both experiments and we did not observe activation in parietal areas such as the caudal IPS known to subserve attention (Wojciulik and Kanwisher, 1999). Third, and perhaps most importantly, if general attention played a role, it should have affected activation equally across all passive viewing conditions. However, if we are indeed measuring attention processing selective for objects within reach, then it is a unique type of attention reflecting a potential for action on reachable objects. Last, although the starting position of the hand (or tool) across trials could arguably attract more attention for closer locations, in which case enhanced responses would be expected for the object nearest the hand (in experiment $1, \mathrm{Near}_{\mathrm{pv}}>$ Medium $_{\mathrm{pv}}$; in experiment $2, \mathrm{NL}_{\mathrm{pv}}$ is higher than the other locations during passive viewing for both hand and tool runs), this prediction was not borne out in the data.

The addition of a tool showed little effect upon the preferred locations in SPOC; nevertheless, this finding shows that that
SPOC codes object reachability with respect to the real distance of the arm and that this coding is resistant to artificial extensions of the hand. Several possible interpretations for this finding are presented in the supplemental material, available at www. jneurosci.org.

\section{SPOC in macaques and humans}

For areas involved in reaching, homologies between the human and macaque monkey brain remain speculative (for review, see Culham et al., 2006; Culham et al., 2008). Nevertheless, the most parsimonious explanation is that the human superior parietooccipital sulcus (i.e., SPOC) is functionally similar to the macaque PO sulcus, which contains areas V6 and V6A.

Macaque V6 and V6A were discovered and characterized by Galletti, Fattori, and their colleagues (Galletti et al., 1996) (for review, see Galletti et al., 2003, 2005). V6 is a purely visual area in macaques with a retinotopic organization. V6 sends projections to area V6A, which contains similar visual properties but no retinotopic organization. V6A also visually encodes object attributes relevant for reaching and grasping like object orientation in space and size (for review, see Galletti et al., 2003). Moreover, some neurons in V6A also encode the spatial location of objects in what appears to be head-centered coordinates (Galletti et al., 1993). V6A neurons also respond during visual and nonvisual goaldirected reaching (Galletti et al., 1997; Fattori et al., 2001, 2005) and are modulated by somatosensory stimulation of the arm and shoulder (Breveglieri et al., 2002). Selective lesions to macaque V6A produce deficits in both reaching and grasping (Battaglini et al., 2002). V6A sends strong output to dorsal premotor cortex (Galletti et al., 1999). In sum, V6A is thought to play a critical role in the visual guidance of reaching and transformations from retinal to spatiomotor coordinate systems. Perhaps one component of such transformations is the determination of whether objects are within reach.

The location of putative V6A in humans, pV6A, is somewhat uncertain at present. A number of reports have speculated that pV6A is in the vicinity of the parieto-occipital sulcus; however, there has been considerable variability in the specific location. Human pV6A has been proposed to lie near the junction of the POS and calcarine sulcus (Ino et al., 2002; Dechent and Frahm, 2003; Bristow et al., 2005; Stiers et al., 2006), in the superior POS (Portin et al., 1998; Pitzalis et al., 2006a; Quinlan and Culham, 2007), and somewhat lateral to the POS (Grol et al., 2007; Verhagen et al., 2008). Localization is complicated by the fact that the fundus of the POS is only millimeters from the IPS and many studies only perform group analyses with extensive smoothing, making it difficult to pinpoint the sulcus in which the activation lies. In our view, the most informed case for the locus of pV6A comes from the work of Pitzalis and colleagues (2006b; 2006a), who suggest that the putative human functional equivalents of V6 (pV6) and V6A (pV6A) lie directly posterior and anterior to the superior end of POS, respectively. In support of the conjecture that $\mathrm{pV} 6 \mathrm{~A}$ lies anterior to the superior POS, a growing number of studies find activation there during reaching and pointing tasks (Astafiev et al., 2003; Connolly et al., 2003; Pitzalis et al., 2006a) and damage to that area in optic ataxia patients with reaching deficits (Karnath and Perenin, 2005; Clavagnier et al., 2007; Himmelbach et al., 2009).

In both our experiments, SPOC activation in individual subjects was typically located in the POS, within $\sim 20 \mathrm{~mm}$ of the superior end of the sulcus, occasionally slightly anterior or posterior to the sulcus. Activation for reaching has also been reported anterior and posterior to the POS [for review, see Culham et al. 
(2008), their Fig. 4]. Although other work from our lab has found SPOC activation for arm transport (Cavina-Pratesi et al., 2006b), grasping versus reaching (Cavina-Pratesi et al., 2007; Króliczak et al., 2008), and near vergence (Quinlan and Culham, 2007), these studies tested different participants, making interstudy comparisons difficult.

In experiment 2, group RFX analyses interestingly showed bilateral activation in the precuneus, anterior to SPOC. This area has been implicated in pointing actions to contralateral targets (Connolly et al., 2003; Fernandez-Ruiz et al., 2007; Zettel et al., 2007) as well as executed, observed and imagined reaching (Filimon et al., 2007). The precuneus has been suggested as a possible human homolog of MIP/PRR (Connolly et al., 2003; FernandezRuiz et al., 2007) (but see Grefkes et al., 2004), which like V6A is involved in reaching actions in macaques (Andersen et al., 1997; Snyder et al., 1997; Andersen and Buneo, 2002).

\section{Conclusions}

In summary, we showed that a reach-related region within SPOC has heightened activation when subjects passively view reachable objects. The response in SPOC is consistent with the hypothesis that it encodes not only actual reaches but also the potential to reach an object with the hand.

\section{References}

Andersen RA (1997) Neural mechanisms of visual motion perception in primates. Neuron 18:865-872.

Andersen RA, Buneo CA (2002) Intentional maps in posterior parietal cortex. Ann Rev Neurosci 25:189-220.

Andersen RA, Snyder LH, Bradley DC, Xing J (1997) Multimodal representation of space in the posterior parietal cortex and its use in planning movements. Ann Rev Neurosci 20:303-330.

Astafiev SV, Shulman GL, Stanley CM, Snyder AZ, Van Essen DC, Corbetta M (2003) Functional organization of human intraparietal and frontal cortex for attending, looking, and pointing. J Neurosci 23:4689-4699.

Battaglini PP, Muzur A, Galletti C, Skrap M, Brovelli A, Fattori P (2002) Effects of lesions to area V6A in monkeys. Exp Brain Res 144:419-422.

Berti A, Frassinetti F (2000) When far becomes near: remapping of space by tool use. J Cogn Neurosci 12:415-420.

Boynton GM, Engel SA, Glover GH, Heeger DJ (1996) Linear systems analysis of functional magnetic resonance imaging in human V1. J Neurosci 16:4207-4221.

Breveglieri R, Kutz DF, Fattori P, Gamberini M, Galletti C (2002) Somatosensory cells in the parieto-occipital area V6A of the macaque. Neuroreport 13:2113-2116.

Bristow D, Frith C, Rees G (2005) Two distinct neural effects of blinking on human visual processing. Neuroimage 27:136-145.

Cavina-Pratesi C, Galletti C, Fattori P, Quinlan DJ, Goodale M, Culham J (2006a) Dissociating the neural correlates of the transport and grip components of reach-to-grasp actions: evidence from event-related fMRI. Paper presented at the Symposium on Cortical Control of Higher Motor Cognition, Lübeck, Germany, May.

Cavina-Pratesi C, Galletti C, Fattori P, Quinlan DJ, Goodale MA, Culham JC (2006b) Event-related fMRI reveals a dissociation in the parietal lobes between transport and grip components in reach-to-grasp movements. Soc Neurosci Abstr 32:307.12.

Cavina-Pratesi C, Goodale MA, Culham JC (2007) FMRI reveals a dissociation between grasping and perceiving the size of real 3D objects. PLoS ONE 2:e424.

Chan JL, Ross ED (1997) Alien hand syndrome: influence of neglect on the clinical presentation of frontal and callosal variants. Cortex 33:287-299.

Clavagnier S, Prado J, Kennedy H, Perenin MT (2007) How humans reach: distinct cortical systems for central and peripheral vision. Neuroscientist 13:22-27.

Colby CL, Duhamel JR (1991) Heterogeneity of extrastriate visual areas and multiple parietal areas in the macaque monkey. Neuropsychologia 29:517-537.

Connolly JD, Andersen RA, Goodale MA (2003) FMRI evidence for a 'parietal reach region' in the human brain. Exp Brain Res 153:140-145.
Culham J, Gallivan JP, Cavina-Pratesi C, Quinlan DJ, eds (2008) fMRI investigations of reaching and ego space in human superior parietal occipito-cortex. Mahwah, NJ: Erlbaum.

Culham JC (2006) Functional neuroimaging: experimental design and analysis. In: Handbook of functional neuroimaging of cognition, Ed 2 (Cabeza R, Kingstone A, eds), pp 53-82. Cambridge, MA: MIT.

Culham JC, Brandt SA, Cavanagh P, Kanwisher NG, Dale AM, Tootell RB (1998) Cortical fMRI activation produced by attentive tracking of moving targets. J Neurophysiol 80:2657-2670.

Culham JC, Danckert SL, DeSouza JF, Gati JS, Menon RS, Goodale MA (2003) Visually guided grasping produces fMRI activation in dorsal but not ventral stream brain areas. Exp Brain Res 153:180-189.

Culham JC, Cavina-Pratesi C, Singhal A (2006) The role of parietal cortex in visuomotor control: what have we learned from neuroimaging? Neuropsychologia 44:2668-2684.

Damasio H (1995) Human brain anatomy in computerized images. Oxford: Oxford UP.

Dechent P, Frahm J (2003) Characterization of the human visual V6 complex by functional magnetic resonance imaging. Eur J Neurosci 17:2201-2211.

de Jong BM, van der Graaf FH, Paans AM (2001) Brain activation related to the representations of external space and body scheme in visuomotor control. Neuroimage 14:1128-1135.

Della Sala S, Marchetti C, Spinnler H (1991) Right-sided anarchic (alien) hand: a longitudinal study. Neuropsychologia 29:1113-1127.

DeSouza JF, Dukelow SP, Gati JS, Menon RS, Andersen RA, Vilis T (2000) Eye position signal modulates a human parietal pointing region during memory-guided movements. J Neurosci 20:5835-5840.

di Pellegrino G, Làdavas E, Farné A (1997) Seeing where your hands are. Nature 388:730.

Evangeliou MN, Raos V, Galletti C, Savaki HE (2009) Functional imaging of the parietal cortex during action execution and observation. Cereb Cortex 19:624-639.

Farnè A, Làdavas E (2000) Dynamic size-change of hand peripersonal space following tool use. Neuroreport 11:1645-1649.

Fattori P, Gamberini M, Kutz DF, Galletti C (2001) 'Arm-reaching' neurons in the parietal area V6A of the macaque monkey. Eur J Neurosci 13:2309-2313.

Fattori P, Kutz DF, Breveglieri R, Marzocchi N, Galletti C (2005) Spatial tuning of reaching activity in the medial parieto-occipital cortex (area V6A) of macaque monkey. Eur J Neurosci 22:956-972.

Fernandez-Ruiz J, Goltz HC, DeSouza JF, Vilis T, Crawford JD (2007) Human parietal "reach region" primarily encodes intrinsic visual direction, not extrinsic movement direction, in a visual-motor dissociation task. Cereb Cortex 17:2283-2292.

Filimon F, Nelson JD, Hagler DJ, Sereno MI (2007) Human cortical representations for reaching: mirron neurons for execution, observation, and imagery. Neuroimage 37:1315-1328.

Galletti C, Battaglini PP, Fattori P (1993) Parietal neurons encoding spatial locations in craniotopic coordinates. Exp Brain Res 96:221-229.

Galletti C, Fattori P, Battaglini PP, Shipp S, Zeki S (1996) Functional demarcation of a border between areas V6 and V6A in the superior parietal gyrus of the macaque monkey. Eur J Neurosci 8:30-52.

Galletti C, Fattori P, Kutz DF, Battaglini PP (1997) Arm movement-related neurons in the visual area V6A of the macaque superior parietal lobule. Eur J Neurosci 9:410-413.

Galletti C, Fattori P, Kutz DF, Gamberini M (1999) Brain location and visual topography of cortical area V6A in the macaque monkey. Eur J Neurosci 11:575-582.

Galletti C, Kutz DF, Gamberini M, Breveglieri R, Fattori P (2003) Role of the medial parieto-occipital cortex in the control of reaching and grasping movements. Exp Brain Res 153:158-170.

Galletti C, Gamberini M, Kutz DF, Baldinotti I, Fattori P (2005) The relationship between V6 and PO in macaque extrastriate cortex. Eur J Neurosci 21:959-970.

Gentilucci M, Roy AC, Stefanini S (2004) Grasping an object naturally or with a tool: are these tasks guided by a common motor representation? Exp Brain Res 157:496-506.

Gibson JJ (1979) The ecological approach to visual perception. Boston: Houghton Mifflin.

Grefkes C, Ritzl A, Zilles K, Fink GR (2004) Human medial intraparietal 
cortex subserves visuomotor coordinate transformation. Neuroimage 23:1494-1506.

Grol MJ, Majdandzić J, Stephan KE, Verhagen L, Dijkerman C, Bekkering H, Verstraten F, Toni I (2007) Parieto-frontal connectivity during visually guided grasping. J Neurosci 27:11877-11887.

Halligan PW, Marshall JC (1991) Left neglect for near but not far space in man. Nature 350:498-500.

Hamilton AF, Grafton ST (2006) Goal representation in human anterior intraparietal sulcus. J Neurosci 26:1133-1137.

Handy TC, Grafton ST, Shroff NM, Ketay S, Gazzaniga MS (2003) Graspable objects grab attention when the potential for action is recognized. Nat Neurosci 6:421-427.

Himmelbach M, Erb M, Klockgether T, Moskau S, Karnath HO (2009) fMRI of global visual perception in simultanagnosia. Neuropsychologia 47:1173-1177.

Holmes NP, Calvert GA, Spence C (2004) Extending or projecting peripersonal space with tools? Multisensory interactions highlight only the distal and proximal ends of tools. Neurosci Lett 372:62-67.

Holmes NP, Sanabria D, Calvert GA, Spence C (2007) Tool-use: capturing multisensory spatial attention or extending multisensory peripersonal space? Cortex 43:469-489.

Ino T, Inoue Y, Kage M, Hirose S, Kimura T, Fukuyama H (2002) Mental navigation in humans is processed in the anterior bank of the parietooccipital sulcus. Neurosci Lett 322:182-186.

Iriki A, Tanaka M, Iwamura Y (1996) Coding of modified body schema during tool use by macaque postcentral neurones. Neuroreport 7:2325-2330.

Karnath HO, Perenin MT (2005) Cortical control of visually guided reaching: evidence from patients with optic ataxia. Cereb Cortex 15:1561-1569.

Klassen LM, Menon RS (2004) Robust automated shimming technique using arbitrary mapping acquisition parameters (RASTAMAP). Magn Reson Med 51:881-887.

Króliczak G, Cavina-Pratesi C, Goodman DA, Culham JC (2007) What does the brain do when you fake it? An fMRI study of pantomimed and real grasping. J Neurophysiol 97:2410-2422.

Króliczak G, McAdam TD, Quinlan DJ, Culham J (2008) The human dorsal stream adapts to real actions and 3D shape processing: a functional magnetic resonance imaging study. J Neurophysiol 100:2627-2639.

Kwong KK, Belliveau JW, Chesler DA, Goldberg IE, Weisskoff RM, Poncelet BP, Kennedy DN, Hoppel BE, Cohen MS, Turner R, Cheng H-M, Brady TJ, Rosen BR (1992) Dynamic magnetic resonance imaging of human brain activity during primary sensory stimulation. Proc Natl Acad Sci U S A 89:5675-5679.

Làdavas E, Zeloni G, Farnè A (1998a) Visual peripersonal space centred on the face in humans. Brain 121:2317-2326.

Làdavas E, di Pellegrino G, Farnè A, Zeloni G (1998b) Neuropsychological evidence of an integrated visuotactile representation of peripersonal space in humans. J Cogn Neurosci 10:581-589.

Làdavas E, Farnè A, Zeloni G, di Pellegrino G (2000) Seeing or not seeing where your hands are. Exp Brain Res 131:458-467.

L'Hermitte F (1983) Utilization behaviour and its relation to lesions of the frontal lobes. Brain 106:237-255.

Makin TR, Holmes NP, Zohary E (2007) Is that near my hand? Multisensory representation of peripersonal space in human intraparietal sulcus. J Neurosci 27:731-740.

Maravita A, Iriki A (2004) Tools for the body (schema). Trends Cogn Sci 8:79-86.

Matelli M, Govoni P, Galletti C, Kutz DF, Luppino G (1998) Superior area 6 afferents from the superior parietal lobule in the macaque monkey. J Comp Neurol 402:327-352.

Medendorp WP, Goltz HC, Crawford JD, Vilis T (2005) Integration of target and effector information in human posterior parietal cortex for the planning of action. J Neurophysiol 93:954-962.

Ogawa S, Tank DW, Menon R, Ellermann JM, Kim SG, Merkle H, Ugurbil K
(1992) Intrinsic signal changes accompanying sensory stimulation: functional brain mapping with magnetic resonance imaging. Proc Natl Acad Sci U S A 89:5951-5955.

Pellijeff A, Bonilha L, Morgan PS, McKenzie K, Jackson SR (2006) Parietal updating of limb posture: an event-related fMRI study. Neuropsychologia 44:2685-2690.

Pitzalis S, Sereno M, Committeri G, Galati G, Fattori P, Galletti C (2006a) A possible human homologue of the macaque V6A. J Vis 6:536a.

Pitzalis S, Galletti C, Huang RS, Patria F, Committeri G, Galati G, Fattori P, Sereno MI (2006b) Wide-field retinotopy defines human cortical visual area v6. J Neurosci 26:7962-7973.

Portin K, Salenius S, Salmelin R, Hari R (1998) Activation of the human occipital and parietal cortex by pattern and luminance stimuli: neuromagnetic measurements. Cereb Cortex 8:253-260.

Prado J, Clavagnier S, Otzenberger H, Scheiber C, Kennedy H, Perenin MT (2005) Two cortical systems for reaching in central and peripheral vision. Neuron 48:849-858.

Previc FH (1998) The neuropsychology of 3-D space. Psychol Bull 124:123-164.

Quinlan DJ, Culham JC (2007) fMRI reveals a preference for near viewing in the human parieto-occipital cortex. Neuroimage 36:167-187.

Rizzolatti G, Gentilucci M, Matelli M (1985) Selective spatial attention: one center, one circuit or many circuits?. In: Attention and performance: mechanisms of attention (Posner MI, Marin O, eds), pp 251-265. Hillsdale, NJ: Lawrence Erlbaum Associates.

Sakata H, Taira M, Mine S, Murata A (1992) Hand-movement-related neurons of the posterior parietal cortex of the monkey: their role in the visual guidance of hand movements. In: Control of arm movement in space: neurophysiological and computational approaches (Caminiti R, Johnson PB, Burnod Y, eds), pp 185-198. Heidelberg: Springer.

Sakata H, Taira M, Murata A, Mine S (1995) Neural mechanisms of visual guidance of hand action in the parietal cortex of the monkey. Cereb Cortex 5:429-438.

Scherberger H, Andersen RA (2007) Target selection signals for arm reaching in the posterior parietal cortex. J Neurosci 27:2001-2012.

Shipp S, Blanton M, Zeki S (1998) A visual somatomotor pathway through superior parietal cortex in the macaque monkey: cortical connections of V6 and V6A. Eur J Neurosci 10:3171-3193.

Snyder LH, Batista AP, Andersen RA (1997) Coding of intention in the posterior parietal cortex. Nature 386:167-170.

Snyder LH, Batista AP, Andersen RA (2000) Intention-related activity in the posterior parietal cortex: a review. Vis Res 40:1433-1441.

Stiers P, Peeters R, Lagae L, Van Hecke P, Sunaert S (2006) Mapping multiple visual areas in the human brain with a short fMRI sequence. Neuroimage 29:74-89.

Talairach J, Tournoux P (1988) Co-planar stereotaxic atlas of the human brain. New York: Thieme Medical.

Umilta MA, Escola L, Instkirvell I, Grammont F, Rochat M, Caruana F, Jezzini A, Gallese V, Rizzolatti G (2008) When pliers become fingers in the monkey motor system. Proc Natl Acad Sci U S A 105:2209-2213.

Verhagen L, Dijkerman HC, Grol MJ, Toni I (2008) Perceptuo-motor interactions during prehension movements. J Neurosci 28:4726-4735.

Verstraten FA, Hooge IT, Culham J, Van Wezel RJ (2001) Systematic eye movements do not account for the perception of motion during attentive tracking. Vis Res 41:3505-3511.

Weiss PH, Marshall JC, Wunderlich G, Tellmann L, Halligan PW, Freund HJ, Zilles K, Fink GR (2000) Neural consequences of acting in near versus far space: a physiological basis for clinical dissociations. Brain $123 \mathrm{Pt}$ 12:2531-2541.

Wojciulik E, Kanwisher N (1999) The generality of parietal involvement in visual attention. Neuron 23:747-764.

Zettel JL, Culham JC, Vilis T, Crawford JD (2007) A comparison of saccade and pointing topograhy in the human posterior parietal cortex. Soc Neurosci Abstr 33:508.12. 